On comparing these days with those on which Bishop's ring was stated to have been visible at Zurich, and with the dates, from M. Forel's paper, of abnormal sunset glows seen at Morges, it was found that the successive appearances of the respective phenomena occurred here about twenty days later than they did in Switzerland. Of course, the weather conditions at single stations introduce irregularities, so that the conclusion must be regarded as uncertain, but supposing it to be approximately correct, since the stations used lie nearly in the same latitude, and we can assume that the drift of the elevated dust-clouds was from west to east, their velocity in passing around the globe, from central Europe to the eastern United States, was about 30 miles per hour, or a rate considerably less than that found from trigonometrical measurements to be the velocity of the highest ice-clouds.

In the case of the great Krakatoa eruption in 1883 , the speed of the ash-cloud as it circled the globe from east to west along the equator, and its slow diffusion toward the poles, was determined from the observation of the successive appearances of coloured suns and brilliant sunset glows in different parts of the world, collected by the Royal Society's committee appointed in 1884 . The assistance in solving the problem of atmospheric circulation which a knowledge of the drift of dust ejected into the upper atmosphere by volcanoes situated in the tropics might furnish would certainly justify obtaining all available data bearing on the march of the abnormal sky colours. Mr. Clayton, of this observatory, began the collection of such data some time ago, but was deterred from continuing the work by reason of the difficulty in obtaining definite information. A task of such magnitude belongs properly to a commission possessing the necessary facilities for collecting and discussing the material, so it is hoped that an organisation like the Krakatoa Committee, the admirable report of which was published in 1887 , may undertake the study of the recent and present remarkable sky colorations, probably occasioned by the eruptions in 1902 of the volcanoes in Martinique and St. Vincent. Mass., U.S.A., December ix.

\section{Internal Oscillation in the Waters of Loch Ness.}

I would beg a little space in your columns to direct attention to some of the conclusions which I draw from temperature observations taken last summer in Loch Ness.

Routine observations have been taken at the south-west end of the loch several times a day since the middle of July, and I find that the temperature at any depth between roo and 300 feet changes with time in a markedly periodic fashion, the duration of a period being approximately three days. At about 200 feet the difference between a maximum and a minimum is something like $5^{\circ} \mathrm{F}$. At greater depths the temperature change is less, but of the same period and the same phase. At depths less than 200 feet also the temperature change appears to fall off in magnitude whilst retaining the same period and phase, but here there appear to be other changes more or less obscuring the simple periodic variation. Diagram I gives a few observations at 200 feet.

I conclude from these observations, and others taken at different parts of the loch, that there is an internal oscillation in the waters-an internal seiche, similar to the swinging which may be set up in the interface between oil and water lying the one above the other in a trough. For such a motion we require liquids of different density lying one above the other; in the loch the upper waters being warmer are lighter than the lower strata, and I think it probable that the region where the temperature changes most abruptly acts as a surface of separation, and is comparable with the interface between the oil and water in the simple arrangement just mentioned. In Diagram 2 I have tried to illustrate the motion. The shaded portion is intended to represent the warmer water, and the hard line the region where the temperature changes most abruptly. Rough calculations on the assumption that the swinging is of this nature give the period of the order observed. A very reNO. I 782 , VOL. 69] markable point is the large amplitude of the vibration. At the ends of the loch the isothermal surfaces suffering the greatest displacement may move through as much as 75 feet.

The observations make it probable that this swinging is started by gales and strong winds. Winds produce a slope of the upper isotherms down towards the lee end of the loch, and the stronger the wind the deeper is this effect felt. So that strong winds are able to displace the relatively deep



isotherms in that region where they act as an interface, and on the wind falling the isotherms swing back and continue to swing freely with a natural period.

Unfortunately it is necessary to wait for the return of summer before more observations can be made bearing on the subject, as the waters are now of almost uniform temperature.

I believe I am right in saying that such a phenomenon had up to this never been even suspected by limnologists.

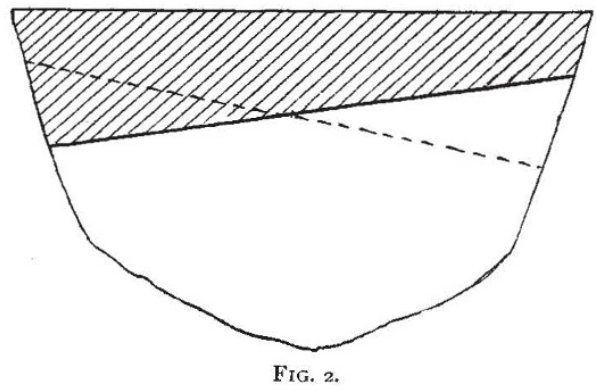

I do not think the temperatures of the deeper strata of water in any lake have been systematically observed. The phenomenon seems to me of great interest and worthy of careful study, as it appears to rank in importance along with the ordinary seiches which have been studied with such care and perseverance by Forel and others.

Scottish Lake Survey, Fort Augustus, N.B., December 